Colloque C3, suppl. au Journal de Physique III, Vol. 1, octobre 1991

\title{
ANALYSE DE LA TRANSITION ENTRE DES DEFORMATIONS ISOTHERMES ET ADIABATIQUES DANS LE CAS DE LA TORSION D'UN TUBE
}

\author{
O. OUSSOUADDI et J.R. KLEPACZKO \\ Université de Metz, Laboratoire de Physique et de Mécanique \\ des Matériaux, URA-CNRS 1215, Ile du Saulcy, F-57045 Metz. \\ France
}

Résumé.Afin d'étudier la transition entre des déformations isothermes et adiabatiques , dans le cas de la torsion d'un tube mince, la technique des différences finies a été appliquée et une série de calcul numérique a été exécutée pour différentes valeurs de la vitesse de déformation nominale de cisaillement, allant de $1 \mathrm{~s}^{-1}$ à $10^{3} \mathrm{~s}^{-1}$. En parallèle nous présentons les effets de la géométrie du tube pour des longueurs allant de $1 \mathrm{~mm}$ à $10 \mathrm{~mm}$. On a trouvé que pour les trois métaux étudiés : l'acier, l'aluminium et le cuivre, la transition se produit pour des vitesses de déformations nominales comprises entre $5 \mathrm{~s}^{-1}$ et $70 \mathrm{~s}^{-1}$. Pour des tubes plus petits, la transition est décalée dans le domaine des gran des vitesses de déformation .

Abstract. In order to study the transition from isothermal to adiabatic conditions of deformtion in a thin tube deformed plastically in torsion at different rates a series of numerical calculations, have been performed. The finite diffrence technique has been applied and calculations were carried out at different nominal shear strain rates within the following limits; $1 \mathrm{~s}^{-1} \leq \Gamma_{\mathrm{n}} \leq 10^{3} \mathrm{~s}^{-1}$. In addition, the effect of the length was studied within the following limits : $2 \mathrm{~mm} \leq \mathrm{L} \leq 10 \mathrm{~mm}$.It has been found that for metals studied, i.e. steel, aluminium and copper, the transition lies in the following limits of the nominal strain rate : $5 \mathrm{~s}^{-1} \leq \Gamma_{\mathrm{n}} \leq 70 \mathrm{~s}^{-1}$. For shorter tubes the transition in shifed into higher strain rates .

\section{INTRODUCTION}

Il est déja établi que , pour des grandes vitesses de déformations, le travail plastique est presque totalement converti en chaleur et que le processus de déformation est adiabatique ; la chaleur produite n'ayant pas le temps de se redistribuer. Dans un autre cas, si les déformations sont appliquées lentement, comme dans le cas d'un chargement quasi-statique , la chaleur est évacuée loin des régions de déformations, et l'éprouvette est essentiellement dans des conditions isothermes .

Quand le processus est adiabatique, Ia chaleur produite peut entrainer un adoucissement thermique, ce qui a pour conséquence, la localisation des déformations plastiques sous forme de bandes de cisaillement adiabatique. Souvent ces bandes sont des zones très étroites de grandes déformations non homogènes, dont le développement est très difficile à prévoir .

L'objet de ce travail est l'étude systématique de la transition isotherme-adiabatique pour plusieurs métaux, dans le cas de la torsion d'un tube, incluant les effets de la vitesse de déformation, de la géométrie de l'éprouvette et de la conduction de la chaleur.

\section{LOIDE COMPORTEMENT ET FORMULATION DU PROBLEME}

Dans un premier temps, la loi de comportement a été identifiée pour l'acier, l'aluminium et le cuivre .Cette loi de comportement est la plus complète utilisée recemment pour l'instabilité adiabatique [ 1] et peut se transcrire sous la forme suivante: [ 2] 


$$
\tau(\Gamma, \Gamma, \mathrm{T})=\mathrm{B}(\mathrm{T})\left(\Gamma_{0}+\Gamma\right)^{\mathrm{n}(\mathrm{T})} \Gamma^{\mathrm{m}(\mathrm{T})}
$$

ou $\tau, \Gamma, \Gamma$ et $T$ sont respectivement la contrainte de cisaillement, la déformation de cisaillement, la vitesse de déformation et la température absolue ; $n(T)$ étant le coefficient d'écrouissage,$m(T)$ le coefficient de sensibilité à la vitesse de déformation, $\mathrm{B}(\mathrm{T})$ le module de plasticité et $\Gamma_{0}$ la déformation initiale,

avec :

$$
\mathrm{m}(\mathrm{T})=\alpha_{\mathrm{m}} \frac{\mathrm{T}}{\mathrm{T}_{\mathrm{m}}}
$$

ou $\quad \alpha_{\mathrm{m}}$ est une constante du matériau, $\mathrm{T}_{\mathrm{m}}$ la température de fusion $(\mathrm{K})$

$$
\mathrm{n}(\mathrm{T})=\mathrm{n}_{0}\left(1 \frac{\mathrm{T}}{\mathrm{T}_{\mathrm{m}}}\right)
$$

ou $n_{0}$ est une constante du matériau

$$
\mathrm{B}(\mathrm{T})=\mathrm{B}_{0} \mathrm{~T}^{-v}
$$

ou $v$ et B sont des constantes du matériau.

Il est important de noter que cette loi de comportement tient compte des effets de la sensibilité à la vitesse de déformation et de la température. L'identification de $B(T), m(T)$ et $n(T)$ a été faite èn utilisant des données expérimentales [ 1 ]

1. Tableau de constantes

\begin{tabular}{|l|lcc|}
\hline \multirow{2}{*}{ CONSTANTES (UNITE) } & \multicolumn{3}{|c|}{ MATERIAU } \\
\cline { 2 - 5 } & Aluminium & Cuivre & Acier \\
\hline$\Gamma_{0}$ & 0.0 & 0.0 & 0.44 \\
\hline$n_{0}$ & 0.5 & 0.6 & 0.215 \\
\hline $\mathrm{Bo}_{0}(\mathrm{MPa})$ & 3857 & 580 & 1092 \\
\hline $\mathrm{T}(\mathrm{K})$ & 933 & 1536 & 1753 \\
\hline $\mathrm{v}_{\mathrm{m}}$ & 0.65 & 0.38 & 0.21 \\
\hline$\alpha{ }_{\mathrm{m}}$ & $5.59810^{-2}$ & $6.0510^{-2}$ & 1 \\
\hline
\end{tabular}

En supposant que la surface latérale du tube est thermiquement isolée, l'équation de conservation de l'énergie conduit à la relation :

$$
\frac{\partial T}{\partial t}=\frac{\kappa(T)}{\rho(T) C_{v}(T)} \Delta T+\frac{1}{\rho(T) C_{v}(T)} f(\tau, \Gamma)
$$

ou $\mathrm{f}(\tau, \Gamma)$ représente la chaleur produite,$\rho(T)$-la densité du matériau, $C_{v}(T)$ - la chaleur spéci fique, $k(T)$ - la conductivité thermique.

Comme la plus part des techniques éxpérimentales, des éssais mécaniques à grandes vitesses de déformations, nous utilisons des éprouvettes tubulaires, ce qui conduit à l'analyse du tube avec les coordonnées cylindriques $(r, \Phi, z)$. Le laplacien en coordonnées cylindriques s'exprime par :

$$
\Delta=\frac{\partial^{2}}{\partial \mathrm{r}^{2}}+\frac{1}{\mathrm{r}} \frac{\partial}{\partial \mathrm{r}}+\frac{1}{\mathrm{r}^{2}} \frac{\partial^{2}}{\partial \Phi^{2}}+\frac{\partial^{2}}{\partial \mathrm{z}^{2}}
$$

Si les propriétés thermiques sont constantes dans l'espace, chaque circonférence d'un élément est déformée identiquement durant l'essai de torsion. Par conséquent la nature axisymétrique du problème nous autorise à ignorer l'angle $\Phi$, de plus le tube étant très mince, on suppose que les variations de température radiale sont négligeables, d'ou l'équation ( 5 ) peut être réecrite sous forme plus explicite: 


$$
\frac{\partial T}{\partial t}=\frac{\kappa(T)}{\rho(T) C_{v}(T)} \frac{\partial^{2} T}{\partial z^{2}}+\frac{1}{\rho(T) C_{v}(T)} \beta \tau(\Gamma, \Gamma, T) \Gamma
$$

ou $\beta$ est le coefficient de Taylor - Quiney qui fixe la proportion de travail plastique converti en chaleur . Les conditions initiales et aux limites suivantes ont été adoptées pour tous les calculs numériques:

(a)_à l'instant initial , la température est constante le long du tube .

$$
\mathrm{T}\left(\mathrm{z}, \mathrm{t}_{0}\right)=\mathrm{T}_{0} \quad, \mathrm{~T}_{0}=300^{\circ} \mathrm{K}
$$

ou $t_{0}$ est l'instant initial, $\mathrm{T}_{0}$ la température initiale .

(b) -- les variations de température aux extrémités du tube sont nulles

$$
\begin{aligned}
& \left(\frac{\partial T}{\partial z}\right)=0 \\
& \left(\frac{\partial T}{\partial z}\right)=0, t \\
& z=L, t
\end{aligned}
$$

Considérons un pas d'espace $\Delta z$, un pas de temps $\Delta t$, et une grille constituée de points $M(i, j)$ de coordonnées $\left(i^{*} \Delta z, j^{*} \Delta t\right)$ dans le plan $(z, t)$. On observe la première et la seconde approximation de la dérivée :

$$
\begin{aligned}
& \frac{\partial T}{\partial t}(i, j)=\frac{1}{\Delta t}\left(T_{i, j}-T_{i, j}\right) \\
& \frac{\partial^{2} T}{\partial z^{2}}(i, j)=\frac{1}{\Delta z^{2}}\left(T_{i-1, j}+2 T_{i, j}+T_{i+1, j}\right)
\end{aligned}
$$

les relations (7), (8), (9) et (10) discrétisées peuvent s'écrire

$$
\begin{aligned}
& \frac{1}{\Delta t}\left(T_{i, j+1}-T_{i, j}\right)=\frac{a^{2}}{\Delta z^{2}}\left(T_{i-1, j}-T_{i, j}+T_{i+1, j}\right)+\frac{\beta}{\rho c_{v}} B_{0} T_{i, j}^{-v}\left(\Gamma_{0}+\Gamma\right)^{n} \Gamma^{m+1} \\
& T(i, 0)=T_{0}-T(1, j)=T(0, j)-T\left(i_{\max ^{-1}, j}\right)=T\left(i_{\max }, j\right)
\end{aligned}
$$

avec :

$$
\mathrm{a}^{2}=\frac{\kappa}{\rho c_{\mathrm{v}}} \quad, \quad \mathrm{T}_{0}=300 \mathrm{~K}
$$

On obtient $T_{i}$ à l'instant $j+1$ comme combinaison de $T_{i-1}, T_{i}, T_{i+1}$ à linstant $j$;la molécule de base est représentée par la fig.i:

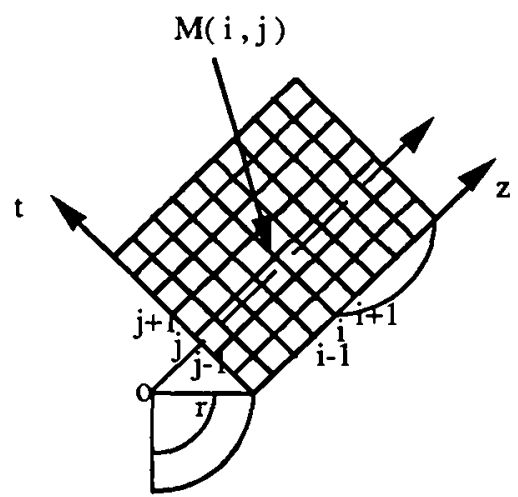

D'ou l'équation (14) devient

Fig.1 Maillage du tube 


$$
T_{i, j+1}=\frac{\Delta t}{\Delta z^{2}} a^{2} T_{i-1, j}+\left(1-\frac{2 \Delta t}{\Delta z} a^{2}\right) T_{i, j}+\frac{\beta}{\rho c_{v}} B_{0}\left(\Gamma_{0}+\Gamma\right)^{n} \Gamma^{m+1} T_{i, j}^{-v}+\frac{\Delta t}{\Delta z^{2}} a T_{i+1, j}
$$

Linconvenient principal de la méthode explicite est qu 'elle nécessite le pas $\Delta t$ suffisament petit si non la résolution de l'équation devient instable et pour que la méthode soit précise il faut que les pas $\Delta t$ et $\Delta z$ soient du même ordre . En effet, il faut que le coefficient de $T_{i, j}$ soit positif , c'est-à-dire :

$$
\frac{\Delta \mathrm{t}}{\Delta \mathrm{z}} \mathrm{a}^{2} \leq \frac{1}{2}
$$

Dans notre cas $\Delta \mathrm{t}=10^{-3}, \Delta \mathrm{z}=10^{-2}$.

Nous résumons dans le tableau ci-dessous les constantes thermiques des matériaux étudiés:

2. Tableau des constantes thermiques

\begin{tabular}{|l|lll|}
\hline \multirow{2}{*}{ CONSTANTE (UNITE) } & \multicolumn{3}{|c|}{ MATERIAU } \\
\cline { 2 - 4 } & Aluminium & Cuivre & Acier \\
\hline$\rho\left(\mathrm{kg} / \mathrm{m}^{3}\right)$ & 2700 & 8390 & 7835 \\
\hline $\mathrm{Cv}(\mathrm{J} / \mathrm{kg} \mathrm{K})$ & 923.8 & 389.7 & 445.4 \\
\hline$\kappa(\mathrm{W} / \mathrm{m} \mathrm{K})$ & 235 & 390 & 65 \\
\hline$\beta$ & 0.9 & 0.9 & 0.9 \\
\hline
\end{tabular}

\section{TRANSITION D'UNE DEFORMATION ISOTHERME A ADIABATIOUE POUR DIFFERENTES LONGUEURS DU TUBE.}

Afin d'étudier cette transition dans le cas de la torsion d'un tube d'épaisseur mince, sollicité à vitesse angulaire constante, on a analysé le procéssus pour plusieurs vitesses de déformations nominales de cisaillement $\Gamma_{n}$, pour cela on a couvert une grande plage de vitesses de déformations allant jusqu'à $10^{3} \mathrm{~s}^{-1}$. En parallèle nous avons étudié l'effet de la taille du tube . Dans tous les cas, les conditions furent imposées pour permettre un transfert de chaleur uniquement par les sections du tube

LONGUEUR L=10 $\mathrm{mm}$

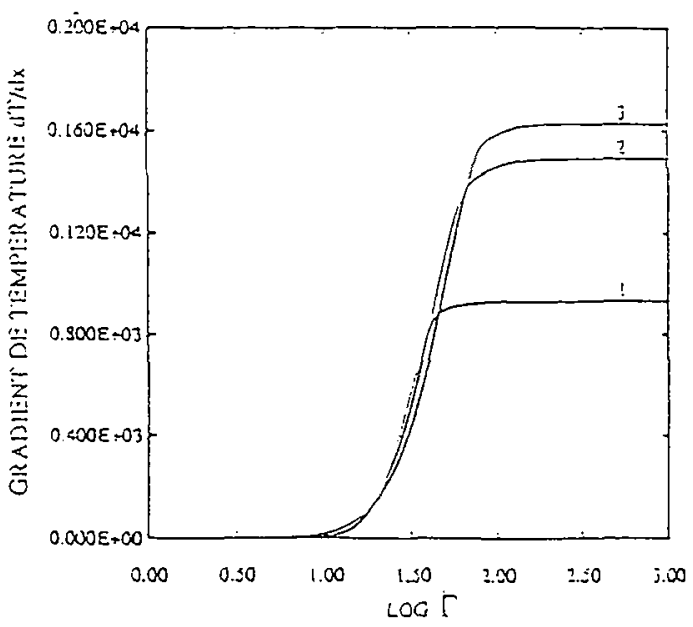

LONGUEUR $L=10 \mathrm{~mm}$

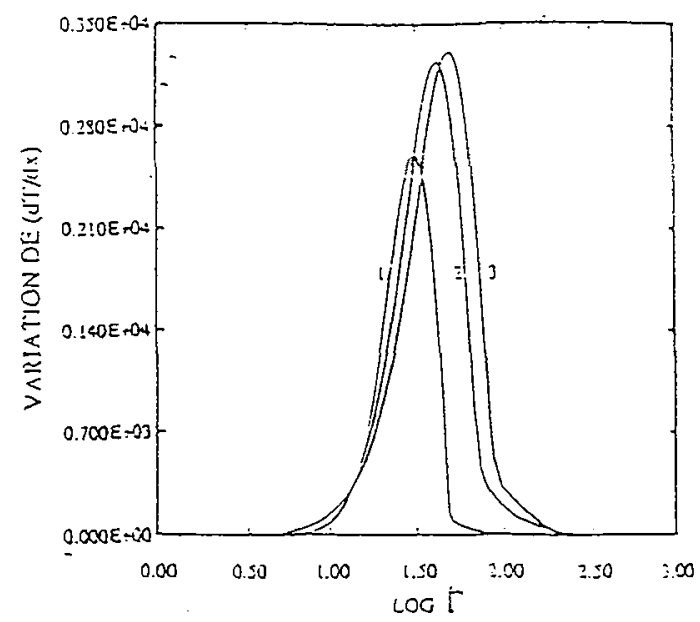

Fig.2 Résultats de calcul numériques de transition à la fonction de la vitesse de déformation nominale pour $\mathrm{L}=10 \mathrm{~mm}$; 1-Acier; 2-Aluminium;3-Cuivre . 
Si la vitesse de déformation nominale est supérieure à $10 \mathrm{~s}^{-1}$, il y a apparition d'une élevation de température $\Delta t$ dûe à la chaleur interne qui augmente. A des vitesses de déformation assez grandes les élevations de températures deviennent véritablement adiabatiques ; pour bien définir la zone de transition nous avons calculé la dérivée du gradient de température par rapport au logarithme de la vi tesse de déformation nominale :

$$
\gamma=\partial\left(\frac{\partial T}{\partial x}\right) / \partial \log \Gamma_{n}
$$

nous obtenons les résultats de la fig. $2 \mathrm{~b}$. qui montre que la zone de transition est formé de deux parties l'une à prédominance isotherme, l'autre à prédominance adiabatique . La valeur de la déformation nominale critique qui délimite le centre de la zone de transition, est de l'ordre de $31 \mathrm{~s}^{-1}$ pour l'acier, de $42 \mathrm{~s}^{-1}$ pour l'aluminium et de $49,5 \mathrm{~s}^{-1}$ pour le cuivre . Les résultats numériques pour les métaux montrent clairement que la zone de transition des déformations isothermes à adiabatiques se trouve entre $\Gamma_{\min }=5 \mathrm{~s}^{-1}$ et $\Gamma_{\max }=70 \mathrm{~s}^{-1}$. A partir de $\Gamma_{\text {nom }}=70 \mathrm{~s}^{-1}$, le procéssus de déformation plastique est entierement adiabatibatique pour l'acier alors que cela nécessite des vitesses de déformations de $180 \mathrm{~s}^{-1}$ pour l'aluminium et de $200 \mathrm{~s}^{-1}$ pour le cuivre. Dans le cas de l'acier , les déforma tions adiabatiques surviennent à partir de vitesses de déformations plus faibes que pour l'aluminium et le cuivre. Car d'une part, la conductivité thermique de l'acier est inférieure à celle de l'aluminium qui est aussi inférieure à celle du cuivre, d'autre part la limite d'élasticité de l'acier est notablement plus élevée que pour l'aluminium et le cuivre.

La fig. 3 montre que ces limites critiques sont décroissantes en fonction de la longueur de l'éprouvette, puisque la zone de transition se trouve entre $10 \mathrm{~s}^{-1}$ et $125 \mathrm{~s}^{-1}$ et d'après la fig. $3 \mathrm{~b}$, la vitesse critique est égale à $36 \mathrm{~s}^{-1}$ pour l'acier, $50 \mathrm{~s}^{-1}$ pour l'aluminium et $59 \mathrm{~s}^{-1}$ pour le cuivre .

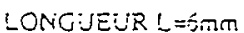

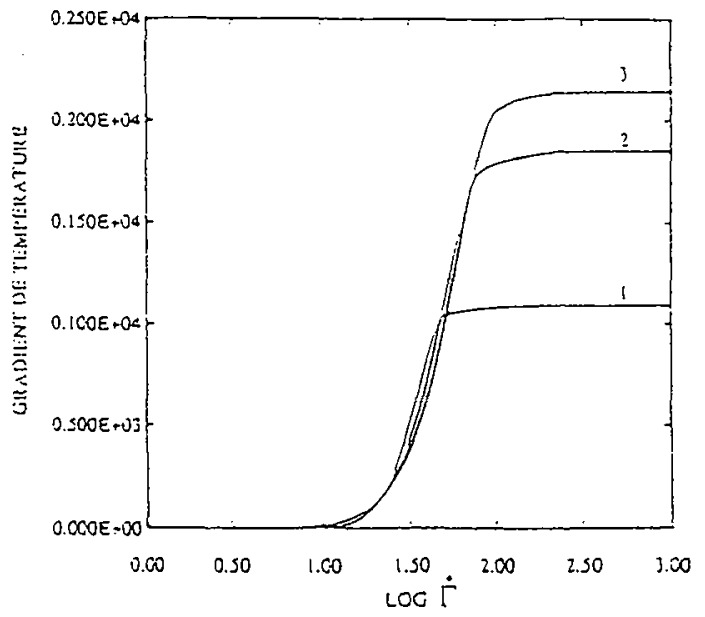

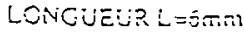

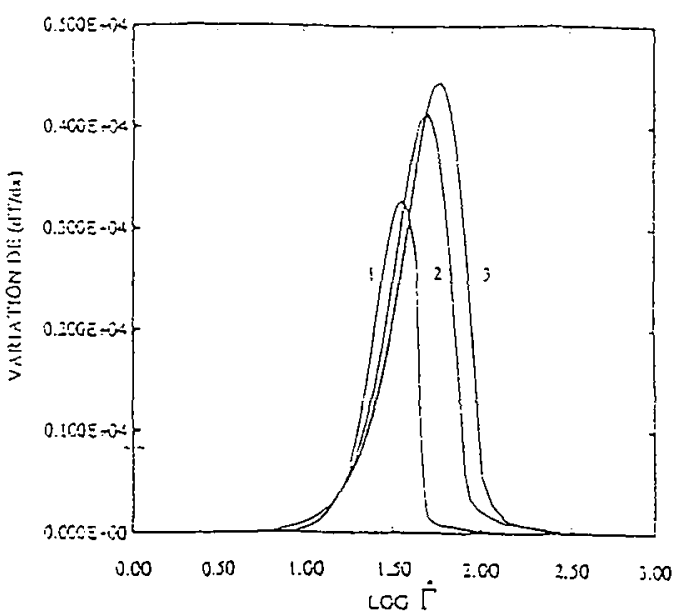

Fig.3 Résultats de calcul numérique de transition à la fonction de la vitsse de déformation nominale pour $\mathrm{L}=6 \mathrm{~mm} ; 1$-Acier ; 2-Aluminium ; 3-cuivre

De même, pour des longueurs très petites, de l'ordre de $2 \mathrm{~mm}$ (fig. 4), la bande de transition survient à partir de plus grandes vitesses de déformation puisque qu'elle est comprise entre $18 \mathrm{~s}^{-1}$ et 177 $\mathrm{s}^{-1}$. Cela s'éxplique par l'évacuation de la chaleur plus importante dans le cas des tubes plus petits . On remarque que plus la longueur est grande, plus la vitesse nominale est plus faible, plus la zone de transition est plus large. Par conséquent, il est impossible d'obtenir des instabilités thermiques pourdes éprouvettes de grandes longueurs et sans imperfection géométrique . Inversement, cela sembe être possible pour des petites longueurs. En effet, d'après les résultats de la fig. 5 cidessous il faut à la formation de bandes de cisaillement et aux instabilités thermiques, à partir des longueurs de l'ordre de $1 \mathrm{~mm}$. 

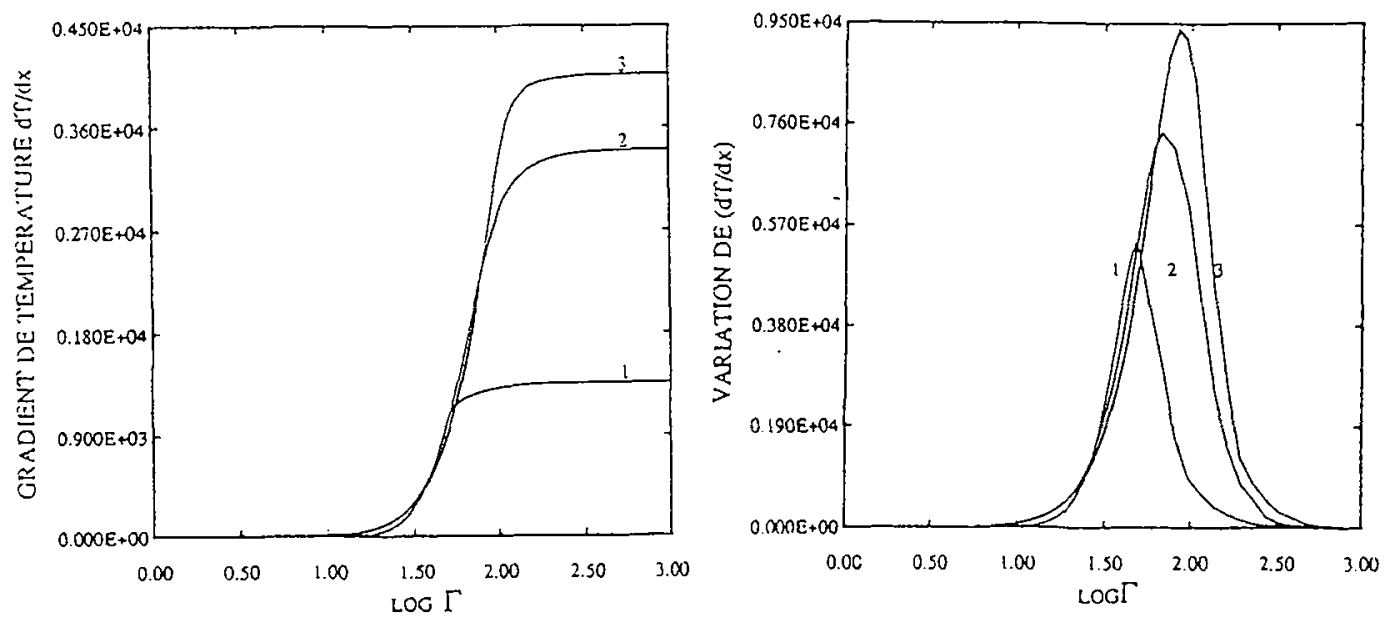

Fig.4 Résultats de calcul numérique de transition à la fonction de la vitesse de déformation nominale pour $\mathrm{L}=2 \mathrm{~mm} ; 1$-Acier ; 2 -Aluminium ; 3 - Cuivre . Nous résumons dans le tableau ci - dessous les valeurs des déformations critiques nominales pour chaque matériau et pour differentes longueurs .

3 . Tableau de vitesses de déformations nominales critiques

\begin{tabular}{|l|l|c|c|}
\cline { 2 - 4 } \multicolumn{1}{c|}{} & \multicolumn{3}{c|}{ Vitesse de défomation nominale critique } \\
\hline longueur (mm) & Acier & Aluminium & Cuivre \\
\hline 1 & 58,8 & 89,0 & 112,0 \\
\hline 2 & 47,5 & 68,5 & 84,5 \\
\hline 4 & 40,5 & 54,0 & 66,0 \\
\hline 6 & 36,0 & 50,0 & 59,0 \\
\hline 10 & 31,0 & 42,0 & 49,0 \\
\hline
\end{tabular}

Afin d'étudier, de facon plus éxacte l'évolution de la vitesse de déformation critque en fonction de la longueur du tube, nous présentons dans la fig.5, les valeurs de $\Gamma_{c r}$ en fonction de $L$.

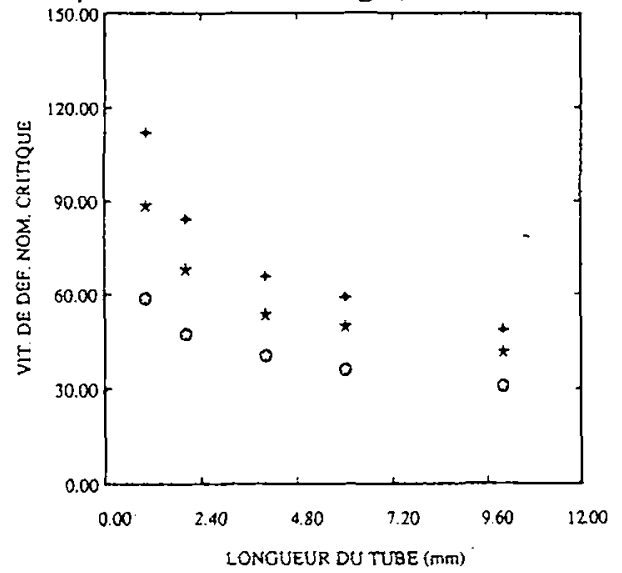

Fig.5 Résultats de calcul numérique $\Gamma_{\mathrm{cr}}(\mathrm{L})$. 
On remarque que pour $L$ comprise entre 1 et $10 \mathrm{~mm}$ la vitesse de déformation nominale critique décroit en fonction de $L$.

Nous présentons dans la fig. 6 les résultats expérimentaux de la compréssion d'une éprouvette cylindrique en cuivre. On observe que la zone de transition est comprise entre $\varepsilon_{\min }=10^{-3} \mathrm{~s}^{-1}$ et $\varepsilon_{\max }=1 \mathrm{~s}^{-1}$. Cette difference entre les résultats numériques et expérìmentaux s'éxplique par le fait qu'il s'agit de la compréssion, donc $\varepsilon=\sqrt{ } 3 \Gamma$ et surtout à cause des frottements au niveau de la section de l'éprouvet te cylindrique ce qui représente une autre source de chaleur en compression .

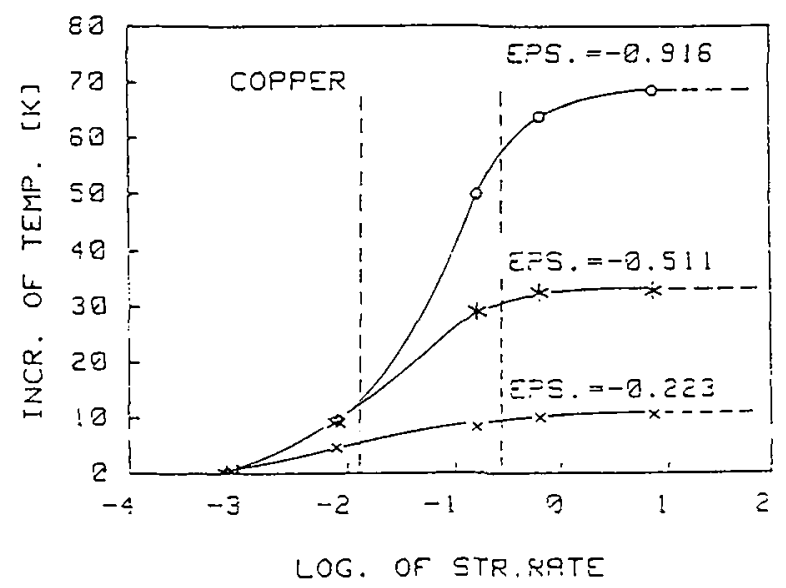

Fig.6 Résultats expérimentaux de compréssion de cuivre d'après [ 3 ]et [ 4 ] pour $\mathrm{L}=37,5 \mathrm{~mm}$.

le contraste entre le comportement des métaux et des polymères est montré dans la fig. 8 ci - dessous qui donne la transition des conditions isothermes à adiabatiques pour le polytereftalen [ 5 ].

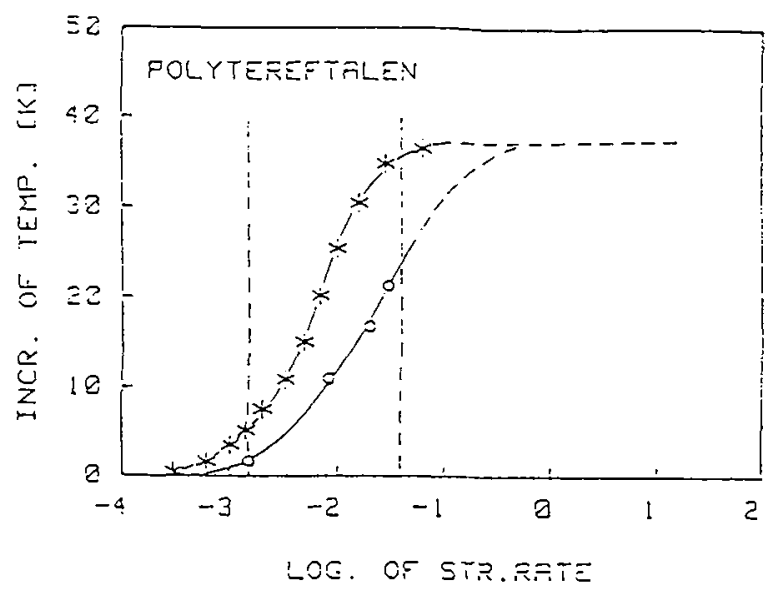

fig.7 Résultats numériques, expérimentaux dans le cas de la tension du polyfteretalen d'après [ 5 ].

Cette analyse a été effectuée en utilisant les données [ 4 ].En conclusion, le seuil de transition survient dans le domaine des vitesses plus faibles pour les polymères et les composites organiques que pour les métaux , ce qui peut s'éxpliquer par leurs faibles chaleur spécifique et conductivité thermique . 


\section{CONCLUSION}

Cette étude nous permet de connaitre avec une bonne précision (tableau 3 ) les trois régions dans le spectre des vitesses de déformations nominales :

-- la première, à faible vitesse de déformation, ou la production de la chaleur peut être négligeable ;

-- la seconde, correspondant à la transition des déformations isothermes à adiabatiques, c'est un cas très important ou il est impératif de considérer et la production et la conduction de la chaleur ;

-- et enfin la région des vitesses de déformations élevées caractérisée par un procéssus purement adiabatique .

Nous avons déterminé également, l'influence de la longueur du tube sur l'apparition des premièrs échauffements et par conséquent, les instabilités ne peuvent se produire que dans le cas des tubes de longueurs très petites, si non il faut introduire des imperfections géométriques .

Dans le cas des éssais de compréssion la production de la chaleur par frottement diminue la valeur de la vitesse de déformation nominale critique de transition isotherme-adiabatique .

Et enfin , on indique que la vitesse de déformation nominale critique pour les composites organiques et plastiques est très faible contrairement aux métaux .

\section{REFERENCES}

[ 1 ] Klepaczko, J.R. , J. Mech. Working Technology, 15 (1987) , 143.

[2] Klepaczko, J.R., in : Modelisation numerique et dynamique des materiaux, Publication du LMA , No.124 (Volume 50 iemc anniversaire du LMA ), Marseille (1991), 335.

[ 3 ] Shimizu, T. and Shinozaki, K. , J. Inst. Techn. Mech. 43 (1989), 48 , (en Japonais).

[ 4 ] Hookway, D.C. , J. Text. Inst. , 49 (1958), 292.

[ 5 ] Vincent, P. I. , Polymer, 1 (1960), 7. 